\title{
High resolution spectral models for solar scaled and $\alpha$-enhanced compositions
}

\author{
P. Coelho ${ }^{1,2}$, G. Bruzual ${ }^{3}$, S. Charlot ${ }^{4}$, A. Weiss ${ }^{1}$ and B. Barbuy ${ }^{2}$ \\ ${ }^{1}$ Max-Planck-Institut für Astrophysik, Karl-Schwarzschild-Strasse 1, Garching, Germany \\ ${ }^{2}$ Universidade de São Paulo, IAG, Rua do Matão 1226, São Paulo 05508-900, Brazil \\ ${ }^{3}$ Centro de Investigaciones de Astronomia, Merida, Venezuela \\ ${ }^{4}$ Institut d'Astrophysique, 98bis Bd. Arago, 75014 Paris, France
}

\begin{abstract}
In the last years, observations made by large international consortia made available hundreds of thousands of high-resolution spectra of galaxies in the local and distant Universe. The interpretation of these spectra in terms of stellar ages and metallicities is the key to reconstructing the star formation and chemical enrichment histories of the Universe. However, current spectral models rely on libraries of observed stars in the solar neighborhood, hampering our hability in interpreting populations that had undergone different star formation histories. Although it is available in the literature Lick/IDS model indices with variable chemical mixtures, we were still lacking high resolution spectral energy distribution models. Those models are required if we want to exploit the total amount of information contained in observed galaxy spectra. We present here our work aiming at the development of such models, constructed with a consistent set of synthetic stellar library and new stellar evolution models.
\end{abstract}

Keywords. galaxies: stellar content, galaxies: abundances

\section{Introduction}

To derive the chemical abundance pattern of galaxies at various redshift is a keystone for understanding the star formation history of the Universe. The most promising way for achieving this goal is to use stellar population (SP) models to interpret high quality galaxy spectra. This requires that the models provide a variety of abundance patterns, covering different star formation histories. Lick/IDS model indices with varying chemical patterns are provided in the literature (e.g. Thomas et al. 2003; Tantalo \& Chiosi 2004; Lee \& Worthey 2005). These models combine theoretical response functions (e.g. Tripicco \& Bell 1995) and empirical fitting functions (e.g. Worthey et al. 1994) in order to produce variable abundance patterns. Alternatively, theoretical fitting functions with explicit dependence to the $\alpha / \mathrm{Fe}$ (Barbuy et al. 2003) can be employed to compute $\alpha$-enhanced models (Mendes de Oliveira et al. 2005). By construction, the mentioned methods are limited to model indices that are described by fitting functions. Therefore, widely used high resolution spectral models (e.g. Vazdekis 1999; Bruzual \& Charlot 2003; Le Borgne et al. 2004) were still unable to account for different chemical patterns, remaining locked to the star formation of the solar neighborhood. A way to overcome this limitation is to use $\alpha$-enhanced libraries of synthetic stellar spectra (Brott \& Hauschildt 2005; Coelho et al. 2005; Gustafsson et al. 2003; Malagnini et al. 2005; Munari et al. 2005) as input for the SSP models. This approach provides ways to explore the effect of the abundance variations in any observable, limited only to the resolution of the stellar spectral library and its wavelength coverage. It is also crucial to employ tracks computed with the same abundance patterns, since above approximately half solar metallicity, the $\alpha$-enhancement changes the evolutionary time scales and isochrones in non-negligible ways (e.g. Salaris \& Weiss 1998). We provide here newly computed high resolution spectral models that 
cover both a solar scaled and an $\alpha$-enhanced abundance pattern typical of populations that have undergone rapid chemical enrichment (e.g. massive early-type galaxies).

\section{New high resolution stellar population models}

Single stellar populations (SSP) models were computed with the GALAXEV evolutionary synthesis code (BC03). Evolutionary tracks were calculated using the Garching Stellar Evolution Code (Weiss \& Schlattl 2000), employing new $\alpha$-enhanced opacity tables (Weiss et al. this conference). The base of our stellar ingredient is the synthetic library by Coelho et al. (2005). This library was extended to cover cool giants employing the model atmospheres by Plez (1992), and corrections were applied to the flux distributions in order to improve the library spectrophotometry. It is worth emphasizing that the same assumptions for the $\alpha$-enhanced mixture was adopted for all main ingredients, providing a highly consistent assessment of the $\alpha$-enhancement effect. Modeling of CN mixing is not included in the current version of the models. The major concern when using synthetic libraries as ingredients for high resolution SSP models is to know whether the synthetic library can safely replace an empirical one. The other side of the worry is that the empirical libraries carry to the model SSP the (sometimes large) uncertainties on the atmospheric parameters of the stars. Even though there is certainly room for improvements in modern synthetic libraries (see Martins; Gustafsson; Peterson; this conference), some tests performed for the Stellar Population Challenge (S. Trager, this conference) show that in full spectrum analysis (Cid Fernandes et al. 2005, STARLIGHT code) these libraries produce results that are not significantly different than when SSP models built with empirical libraries are employed. Main discrepancies are found to be regions either dominated by Balmer lines or highly sensitive to $\mathrm{C}$ and $\mathrm{N}$ abundances. Even then, the age and metallicity derived from the whole spectrum agree with empirical libraries in $\sim 1$ Gyr and $\sim 0.13$ dex respectively. Our SSP models were computed for three values of iron abundance $(-0.5,0.0$ and $0.2 \mathrm{dex})$, for both solar scaled and an $\alpha$ enhanced pattern (overall metallicities $0.005<\mathrm{Z}<0.048$ ). Ages range from 3 to 15 Gyr. The wavelength coverage is 300 to $1340 \mathrm{~nm}$ with a resolution of $\mathrm{FWHM}=1 \AA$ (sampling of $0.2 \AA$ ). The $\alpha$-enhancement is considered to be a flat enhancement of 0.4 dex for all classical $\alpha$-elements: O, Ne, Mg, Si, S, Ca and Ti. This is the same $\alpha$-enhanced mixture adopted in the synthetic stellar high resolution spectra, in the model atmospheres, and in the new evolutionary tracks.

\section{Results}

In order to test our models with observations, we selected galaxies from the SDSS-DR4 that are representative of massive early-type populations. Only galaxies with concentration parameter $\mathrm{c}>2.8, \mathrm{~S} / \mathrm{N} \geqslant 40$ and velocity dispersions between 250 and $300 \mathrm{~km} / \mathrm{s}$ were selected. The models were convolved to the instrumental resolution of the SDSS spectrograph, and then broadened to a velocity dispersion of $275 \mathrm{~km} / \mathrm{s}$. We found that all indices that respond strongly to $\mathrm{Fe}$ and $\alpha$-elements are very well reproduced, and some comparisons between observations and models are given in Figure 1. In Figure 2 we illustrate another application of the models: to assess the effect of $\alpha$-enhancement on broad band colours. The $\alpha$-enhanced colours U-B, B-V and $\mathrm{u}-\mathrm{g}$ are bluer than solar-scaled ones at fixed $[\mathrm{Fe} / \mathrm{H}]$, in agreement with the work by Cassisi et al. (2004) on $\alpha$-enhanced colour transformations. 


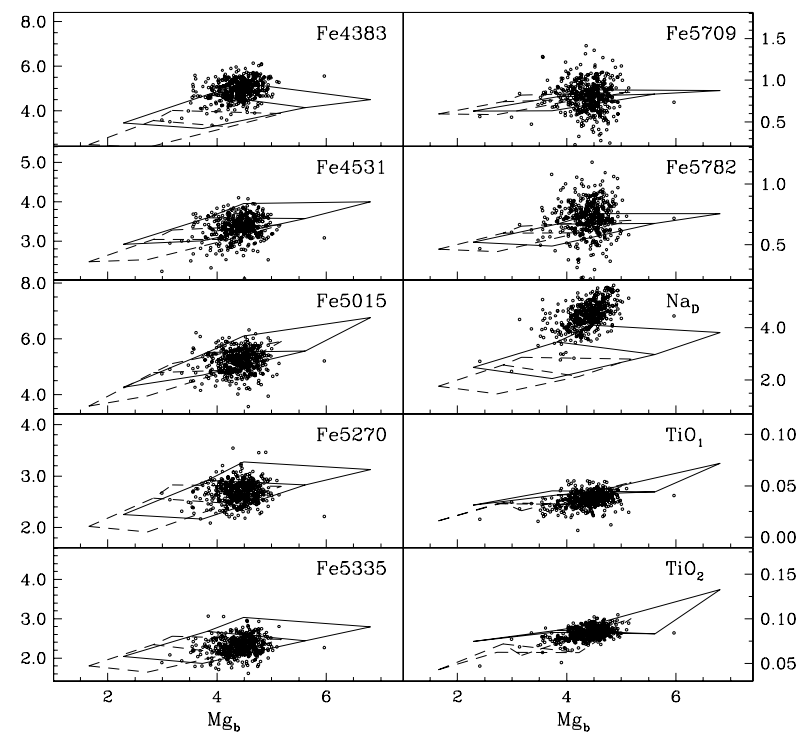

Figure 1. Comparison between our models (lines) and elliptical galaxies from SDSS-DR4 (points) for some Lick/IDS indices. Models for 12Gyr and 4 Gyrs are shown as solid and dashed lines respectively. Models show two values of $[\alpha / \mathrm{Fe}]\left(0.0\right.$ and 0.4 ; lower and higher $\mathrm{Mg}_{b}$ values respectively) and three values of $[\mathrm{Fe} / \mathrm{H}](-0.5,0.0,0.2)$.

\section{Conclusions}

We present a new set of high resolution SSP models. These models employ an updated and improved version of the Coelho et al. synthetic stellar library, as well as new stellar evolutionary tracks computed with updated opacities (Weiss et al. this conference). We provide models for an abundance pattern different from that of the solar neighborhood. With these new models, the effect of the $\alpha$-enhancement on a large number of observables can be explored. The present models are well suitable for deriving the response of any observable in the wavelength region covered by the models to the overall $\alpha$-enhancement: broad-band colours, Lick and non-Lick indices, etc. These models can also be employed in full spectrum analysis of stellar populations (e.g. Panter et al. 2003), with the caveat that regions known to have important systematic offsets when compared to empirical stellar libraries should be masked. We compared our spectral predictions with indices measured in elliptical galaxies observed by the SDSS-DR4. All indices highly sensitive to Fe and $\alpha$-elements abundances are well reproduced. All models and ingredients will be available publicly.

\section{Acknowledgements}

PC acknowledges grants from a Fapesp fellowship ( $\left.{ }^{\circ} 2005 / 03840-3\right)$ and the Latin American-European Network on Astrophysics and Cosmology (LENAC) of the European Union's ALFA Programme.

\section{References}

Barbuy, B., Perrin, M.-N., Katz, D., Coelho, P., Cayrel, R., Spite, M., \& Van’t Veer-Menneret, C., 2003, A\&A, 404, 661 


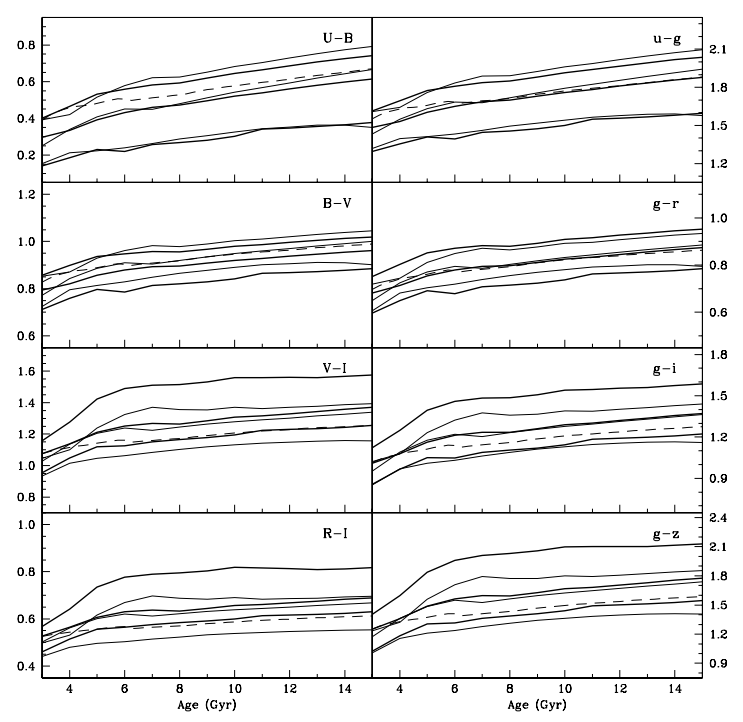

Figure 2. The colour evolution predicted by the present models in Johnson-Cousin (left) and SDSS systems (right). The thin solid lines are the solar-scaled models and the thick solid lines are $\alpha$-enhanced models. The $[\mathrm{Fe} / \mathrm{H}]$ values $(-0.5,0.0$ and 0.2$)$ increase from bottom to top . The dashed line is the solar model by $\mathrm{BC} 03$, for reference.

Brott, I., \& Hauschildt, P. H., 2005, in Turon C., O'Flaherty K. S., Perryman M. A. C., eds, ESA SP-576: The Three-Dimensional Universe with Gaia A PHOENIX Model Atmosphere Grid for Gaia. p. 565

Bruzual, G., \& Charlot, S., 2003, MNRAS, 344, 1000

Cassisi, S., Salaris, M., Castelli, F., \& Pietrinferni, A., 2004, ApJ, 616, 498

Cid Fernandes, R., Mateus, A., Sodré, L., Stasińska, G., \& Gomes, J. M., 2005, MNRAS, 358, 363

Coelho, P., Barbuy, B., Melendez, J., Schiavon, R., \& Castilho, B., 2005, A\&A, 443, 735

Gustafsson, B., Edvardsson, B., Eriksson, K., Jørgensen, U. G., Mizuno-Wiedner, M., \& Plez, B., 2003, in Piskunov N., Weiss W. W., Gray D. F., eds, IAU Symposium A New MARCS Grid. pp 4P-+

Le Borgne, D., Rocca-Volmerange, B., Prugniel, P., Lançon, A., Fioc, M., \& Soubiran, C., 2004, A\&A, 425, 881

Lee, H.-c., \& Worthey, G., 2005, ApJS, 160, 176

Malagnini, M. L., Franchini, M., Morossi, C., \& di Marcantonio, P., 2005, in Turon C., O'Flaherty K. S., Perryman M. A. C., eds, ESA SP-576: The Three-Dimensional Universe with Gaia alpha-Enhancement in Thin and Thick Disc Stars: An Atlas of Synthetic Spectra. pp 595--

Mendes de Oliveira, C., Coelho, P., González, J. J., \& Barbuy, B., 2005, AJ, 130, 55

Munari, U., Sordo, R., Castelli, F., \& Zwitter, T., 2005, A\&A, 442, 1127

Panter, B., Heavens, A. F., \& Jimenez, R., 2003, MNRAS, 343, 1145

Plez B., 1992, A\&AS, 94

Salaris, M., \& Weiss, A., 1998, A\&A, 335, 943

Tantalo, R., \& Chiosi, C., 2004, MNRAS, 353, 917

Thomas, D., Maraston, C., \& Bender, R., 2003, MNRAS, 339, 897

Tripicco, M. J., \& Bell, R. A., 1995, AJ, 110, 3035

Vazdekis, A., 1999, ApJ, 513, 224

Weiss, A., \& Schlattl, H., 2000, A\&A Supplement, 144, 487

Worthey, G., Faber, S. M., Gonzalez, J. J., \& Burstein, D., 1994, ApJS, 94, 687 


\section{Discussion}

Peterson: What line list did you use?

Coelho: The line list has being built by Beatriz's group at Sao Paulo for many years. It is based on laboratory measurements, and a list of sources given in Coelho et al. (2005). But many oscillator strengths and broadening parameters have been fine tunned by many people at different wavelength ranges.

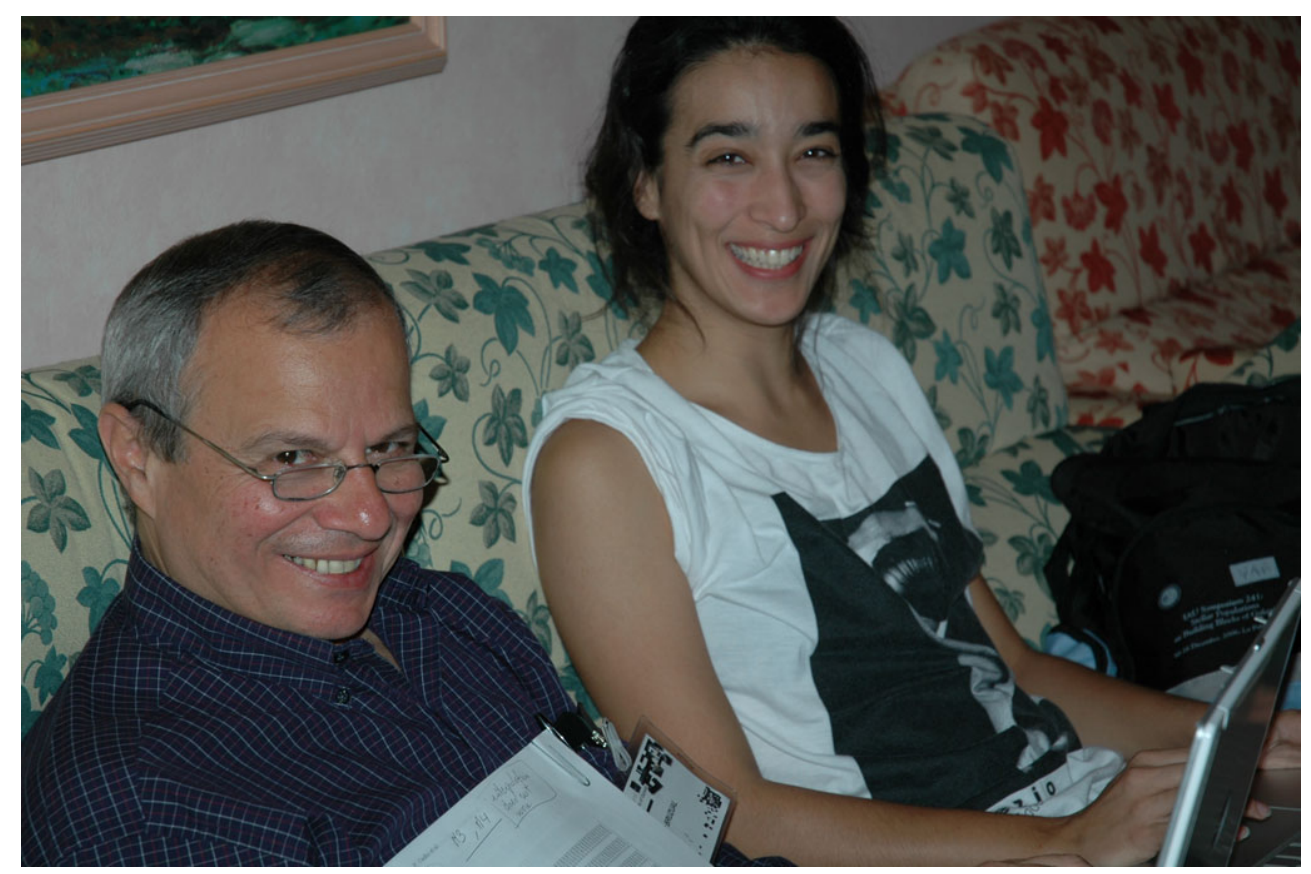

The speaker with Gustavo Bruzual (left). 